\title{
REVIEW
}

\section{Clinically Meaningful Efficacy and Acceptability of Low-Frequency Repetitive Transcranial Magnetic Stimulation (rTMS) for Treating Primary Major Depression: A Meta-Analysis of Randomized, Double-Blind and Sham-Controlled Trials}

\author{
Marcelo T Berlim**, ${ }^{*}$, Frederique Van den Eynde' and Z Jeff Daskalakis ${ }^{3}$ \\ 'Neuromodulation Research Clinic, Douglas Mental Health University Institute and McGill University, Montréal, Québec, Canada; ${ }^{2}$ Depressive \\ Disorders Program, Douglas Mental Health University Institute and McGill University, Montréal, Québec, Canada; ${ }^{3}$ Brain Stimulation Treatment \\ and Research Program, Centre for Addiction and Mental Health and University of Toronto, Ontario, Canada
}

\begin{abstract}
Clinical trials on low-frequency repetitive transcranial magnetic stimulation (LF-rTMS) over the right dorsolateral prefrontal cortex have yielded conflicting evidence concerning its overall efficacy for treating major depression (MD). As this may have been the result of limited statistical power of individual trials, we have carried the present systematic review and meta-analysis to examine this issue. We searched the literature for English language randomized, double-blind and sham-controlled trials (RCTs) on LF-rTMS for treating MD from 1995 through July 2012 using EMBASE, PsycINFO, Cochrane Central Register of Controlled Trials, SCOPUS, and ProQuest Dissertations \& Theses, and from October 2008 until July 2012 using MEDLINE. The main outcome measures were response and remission rates as well as overall dropout rates at study end. We used a random-effects model, odds ratios (ORs) and number needed to treat (NNT). Data were obtained from eight RCTs, totaling 263 subjects with MD. After an average of I $2.6 \pm 3.9$ rTMS sessions, 38.2\% (50/I 3 I) and I 5. I\% (20/I32) of subjects receiving active LF-rTMS and sham rTMS were classified as responders $(\mathrm{OR}=3.35 ; 95 \% \mathrm{Cl}=1.4-8.02 ; p=0.007)$. Also, 34.6\% (35/IOI) and 9.7\% (I0/I03) of subjects receiving active LF-rTMS and sham rTMS were classified as remitters (OR=4.76; $95 \% \mathrm{Cl}=2.13-10.64 ; p<0.000 \mathrm{I})$. The associated NNT for both response and remission rates was 5. Sensitivity analyses have shown that protocols delivering > 1200 magnetic pulses in total as well as those offering rTMS as a monotherapy for MD were associated with higher rates of response to treatment. No differences on mean baseline depression scores and dropout rates for active and sham $r$ TMS groups were found. Finally, the risk of publication bias was low. In conclusion, LF-rTMS is a promising treatment for MD, as it provides clinically meaningful benefits that are comparable to those of standard antidepressants and high-frequency rTMS. Furthermore, LF-rTMS seems to be an acceptable intervention for depressed subjects.
\end{abstract}

Neuropsychopharmacology (2013) 38, 543-55I; doi:I0.1038/npp.2012.237; published online 19 December 2012

Keywords: depression (unipolar/bipolar); psychiatry \& behavioral sciences; neurophysiology; mood/anxiety/stress disorders; transcranial magnetic stimulation; meta-analysis

\section{INTRODUCTION}

Repetitive transcranial magnetic stimulation (rTMS) is a non-invasive technique for modulating cortical and subcortical function with the use of rapidly changing electromagnetic fields generated by a coil placed over the scalp (George and Post, 2011). Depending on the parameters of stimulation, rTMS can modulate cortical excitability in

*Correspondence: Dr MT Berlim, Douglas Mental Health University Institute, 6875 LaSalle Boulevard, FBC-3 Pavilion, Montréal, Québec H4H IR3, Canada. E-mail: nrc.douglas@me.com

Received 22 August 2012; revised 12 November 2012; accepted 13 November 2012; accepted article preview online 19 November 2012 relatively focal areas (Fregni and Pascual-Leone, 2007), with frequencies $\leqslant 1 \mathrm{~Hz}$ (low-frequency rTMS or LF-rTMS) being usually inhibitory, and higher frequencies $(\geqslant 5 \mathrm{~Hz}$; high-frequency rTMS or HF-rTMS) being usually excitatory (Fitzgerald et al, 2002; Marangell et al, 2007).

rTMS is being increasingly investigated as a potential treatment for several neuropsychiatric disorders (George et al, 2009), and particularly for major depression (MD) (Daskalakis et al, 2008). The first evidence for its antidepressant effects was observed with a high-frequency protocol $(20 \mathrm{~Hz})$ applied to the left dorsolateral prefrontal cortex (DLPFC) (George et al, 1995), and to date several meta-analyses have confirmed the overall efficacy and safety of HF-rTMS for treating depressed subjects (Berlim et al, 
submitted-b; Slotema et al, 2010). As the latter can be uncomfortable at higher intensities (Janicak et al, 2008), and is also associated with an increased risk of adverse effects (Loo et al, 2008), LF-rTMS over the right DLPFC has been more recently proposed as an alternative therapeutic strategy for MD (Schlaepfer et al, 2003). Indeed, initial clinical trials showed that LF-rTMS has antidepressant properties (Klein et al, 1999). However, questions remain as to whether this neuromodulation technique has clinically relevant effects in $\mathrm{MD}$ as RCTs to date have produced conflicting results. For example, Hoppner et al (2003) and Kauffmann et al (2004) showed that LF-rTMS was not superior to sham rTMS, whereas Stern et al (2007) and Pallanti et al (2010) found that LF-rTMS was associated with significantly higher rates of clinical improvement when compared with sham rTMS. A likely reason for these discrepant findings might be the lack of statistical power among some of the individual RCTs (Maxwell et al, 2008). Therefore, the use of meta-analytical approaches could be helpful in examining this issue by allowing the integration of findings from multiple studies and the more accurate estimation of the treatment effects associated with LFrTMS' (Huf et al, 2011).

Recently, Schutter (2010) has conducted the first metaanalysis on LF-rTMS for MD and found that this neuromodulation technique was significantly more effective than sham rTMS in reducing post-treatment scores on standard depression scales (ie, Hedges'g $=0.63$; $95 \% \mathrm{CI}=0.03-1.24)$. However, the clinical magnitude of these reported changes in depressive symptomatology is difficult to interpret, especially in light of current recommendations on the assessment of treatment efficacy in MD that advocate the use of more clinically relevant outcomes such as response and remission rates (Rush et al, 2006a). Furthermore, the study by Schutter (2010) lacked information regarding some of its key methodological aspects (eg, search syntaxes and outputs, reasons for excluding studies), and this poses a limitation to its replicability.

Therefore, to summarize the best available evidence on the use of LF-rTMS for treating MD (taking into consideration the limitations of the previous meta-analysis), we have carried out a systematic review and meta-analysis of randomized, double-blind and sham-controlled trials. Furthermore, we assessed overall treatment acceptability based on the differential dropout rates among subjects receiving active or sham rTMS.

\section{METHODOLOGY OF THE LITERATURE REVIEW}

\section{Search Strategy}

We identified articles for inclusion in this meta-analysis by

- Screening the bibliography of the previous meta-analyses on rTMS for MD (Allan et al, 2011; Burt et al, 2002; Couturier, 2005; Gross et al, 2007; Herrmann and Ebmeier, 2006; Kozel and George, 2002; Lam et al, 2008; Martin et al, 2002; Martin et al, 2003; McNamara et al, 2001; Schutter, 2009; Slotema et al, 2010), of the only meta-analysis on LF-rTMS for MD published to date (Schutter, 2010), as well as of all included RCTs;

- Searching MEDLINE from 1 October 2008 until 22 July 2012 [as previous meta-analyses have screened this database up to late 2008 (Allan et al, 2011; Slotema et al, 2010)];

- Searching EMBASE, PsycINFO, the Cochrane Central Register of Controlled Trials (CENTRAL), SCOPUS and ProQuest Dissertations \& Theses (PQDT) from 1 January 1995 until 22 July 2012;

The search procedures (including syntaxes, parameters, and results) are described in detail in the Supplementary Material.

\section{Study Selection}

Candidate studies (judged on the basis of their title and abstract) had to satisfy the following criteria (Higgins and Green, 2008):

Study Validity. Random allocation; double-blind (ie, patients and clinical raters blinded to treatment conditions); sham-controlled (ie, coil angled on the scalp or use of a specific sham coil); parallel or crossover design (with only data from the initial randomization being used for the latter to avoid carryover effects); $\geqslant 5$ subjects with MD randomized per study arm;

Sample characteristics. Subjects aged 18-75 years with a diagnosis of primary major depressive episode (unipolar or bipolar) according to the Diagnostic and Statistical Manual of Mental Disorders (APA, 1994) or the International Classification of Diseases (WHO, 1992) criteria;

Treatment characteristics. LF-rTMS (ie, $\leqslant 1 \mathrm{~Hz}$ ) given for $\geqslant 10$ sessions either as a monotherapy or as an augmentation strategy for MD;

Publication-related. Articles written in English. Studies were excluded if they:

- Enrolled subjects with 'narrow' diagnoses (eg, postpartum depression) or secondary MD (eg, vascular depression);

- Started rTMS at the same time as a new antidepressant;

- Did not report rates of response to treatment and/or remission.

In cases where potentially eligible studies were missing key data for our meta-analysis, their corresponding authors were contacted twice by E-mail at a 2-week interval (please refer to the Supplementary Material for additional information).

\section{Data Extraction}

Data were recorded in a structured fashion as follows:

Sample characteristics. Mean age, sex, treatment strategy used (ie, augmentation or monotherapy), primary diagnosis, presence of treatment-resistant MD;

rTMS-related. Stimulation frequency and intensity (including the total number of stimuli delivered), number of treatment sessions, type of sham; 
Primary outcome measure. Number of responders (Rush et al, 2006a) to treatment based on the RCTs' primary efficacy measure (defined as a $\geqslant 50 \%$ reduction in posttreatment scores on the Hamilton Depression Rating Scale [HAM-D] (Hamilton, 1960) or on the Montgomery-Asberg Depression Rating Scale [MADRS] (Montgomery and Asberg, 1979)) at study end;

Secondary outcome measure. Number of remitters (Rush et al, 2006a) based on the RCTs' primary efficacy measure (eg, 17- or 21 -item HAM-D scores $\leqslant 7$ or $\leqslant 8$, respectively, or MADRS scores $\leqslant 6$ ) at study end;

Acceptability of treatment. Overall dropout rates of active and sham rTMS groups at study end.

\section{Data Synthesis and Analyses}

Analyses were performed using Comprehensive MetaAnalyses Version 2.0 (Biostat, Englewood, NJ, USA), and IBM SPSS Version 20 (IBM Corporation, Chicago, IL, USA).

We used a random-effects model because it was assumed that the true treatment effects had likely varied between the included RCTs (Riley et al, 2011). If provided, intention-totreat data, using a method such as 'last observation carried forward', were preferred over data from completers (Fergusson et al, 2002). The efficacy of LF-rTMS for MD as well as its acceptability were investigated by odds ratios (ORs) (Deeks, 2002) and the number needed to treat (NNT) for rates of response/remission and dropouts (Borenstein et al, 2009). We considered a NNT $\leqslant 10$ as clinically meaningful because such a treatment difference would be routinely encountered in day-to-day clinical practice (Citrome, 2011). Also, to rule out the presence of baseline differences in depressive symptoms between active and sham rTMS groups, we computed the pooled standardized mean difference (SMD) of subjects' baseline scores on the HAM-D or the MADRS. Moreover, we conducted sensitivity analyses to determine the potential impact of the following variables on the effect size estimates for response and remission: total number of sessions (ie, $10 v s>10$ ) or pulses $(\leqslant 1200 v s>1200)$, percentage of the resting motor threshold $(\leqslant 100$ vs $>100 \%)$, primary diagnosis (ie, samples with unipolar depression only $v s$ samples with mixed unipolar/bipolar depression) and treatment strategy (ie, rTMS as augmentation $v s$ monotherapy).

Heterogeneity was assessed using the $Q$ statistics and $I^{2}$ (Cooper et al, 2009). Values of $p<0.1$ for the former and $>35 \%$ for the latter were deemed as indicative of study heterogeneity (Borenstein et al, 2009). Finally, we used Funnel Plots, Rosenthal's Fail-Safe N (Rosenthal, 1979), Egger's Regression Intercept (Egger et al, 1997), and Duval and Tweedie's Trim \& Fill procedure (Duval and Tweedie, 2000 ) to test for the presence of publication bias (Borenstein et al, 2009; Cooper et al, 2009).

\section{RESULTS}

\section{Literature Search}

Of the 10 RCTs on LF-rTMS for MD included in the previous meta-analyses, sixwere selected for the present

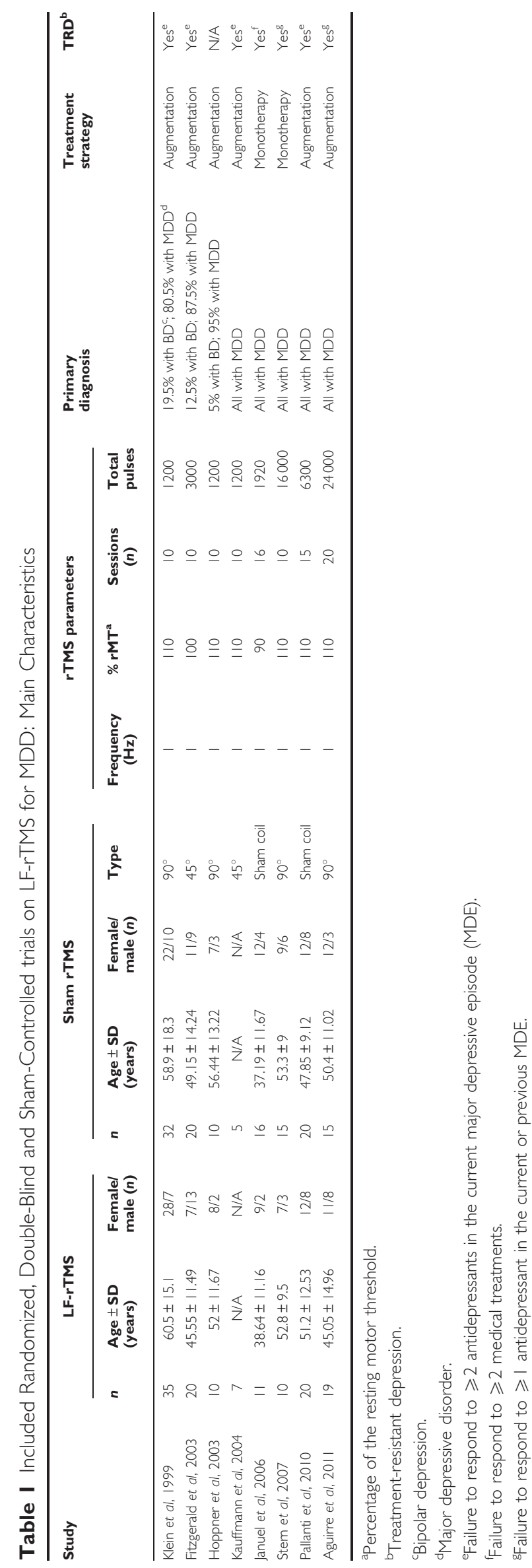

Neuropsychopharmacology 
investigation (Fitzgerald et al, 2003; Hoppner et al, 2003; Januel et al, 2006; Kauffmann et al, 2004; Klein et al, 1999; Stern et al, 2007). Also, we retrieved four RCTs on LF-rTMS for MD from MEDLINE, PsycINFO, EMBASE, CENTRAL, SCOPUS, and PQDT. Of these, only two RCTs met the eligibility criteria (Aguirre et al, 2011; Pallanti et al, 2010), as the remaining only applied LF-rTMS over the left DLPFC (Speer et al, 2009; Speer et al, 2000). Please refer to the Supplementary Material for a detailed description of the study selection procedure.

\section{Included RCTs: Main Characteristics}

Overall, eight RCTs were included in our meta-analysis, totaling 263 subjects with MD, of whom 131 were randomized to active LF-rTMS (mean age $=49.39 \pm 7$ years; $65.6 \%$ females), and 132 were randomized to sham rTMS (mean age $=50.46 \pm 7.07$ years; $66.4 \%$ females). The mean number of rTMS sessions delivered was $12.6 \pm 3.9$. Also, LFrTMS was used as an augmentation strategy for MD in most RCTs (6 out of 8 ), and most subjects had some degree of treatment-resistant depressive illness. The main characteristics of the included RCTs are described in Table 1.

\section{Response Rates}

Data relating to response rates were available from all eight RCTs. Overall, 50 (out of 131; 38.2\%) and 20 (out of 132; $15.1 \%)$ subjects receiving active LF-rTMS or sham rTMS were classified as responders to treatment, respectively. The pooled OR was $3.35(95 \% \mathrm{CI}=1.34-8.02 ; z=2.71$; $p=0.007$ ), indicating a significant difference in outcome favoring active LF-rTMS (Figure 1). The risk difference translated into a NNT of $5(95 \% \mathrm{CI}=3-7.9)$, meaning that about one in every five patients will present with a response following LF-rTMS.

Heterogeneity between RCTs did not exceed that expected by chance $\left(\mathrm{df}=7 ; Q_{7}=10.99, p=0.14 ; I^{2}=34.18\right)$, implying that the variance among the effect sizes was no greater than expected by sampling error. The Fail-Safe $\mathrm{N}$ for response rates was 19 , indicating that at least 19 unpublished or missing null-findings would be needed to render the clinical effect of active LF-rTMS statistically non-significant (ie, $p \geqslant 0.05$ ). Additionally, the associated Funnel Plot was reasonably symmetrical (Figure 2). Publication bias was assessed more conservatively with Egger's regression intercept, which was $0.95 \quad(\mathrm{df}=6 ; t=0.73$; two-tailed $p=0.49$ ), suggesting a low risk of publication bias. Also, no RCT was trimmed in the Duval and Tweedie's Trim \& Fill procedure, thus reinforcing the low risk of publication bias.

\section{Remission Rates}

Data relating to remission rates were available from six RCTs. Overall, significantly more patients receiving active LF-rTMS were classified as remitters as compared with those receiving sham rTMS (34.6\% (35/101) vs 9.7\% (10/ $103)$, respectively). The pooled OR was $4.76(95 \% \mathrm{CI}=2.13-$ $10.64 ; z=3.8 ; p<0.0001$ ) (Figure 3 ). The risk difference translated into a NNT of five $(95 \% \mathrm{CI}=2.8-7.1)$.

Heterogeneity between RCTs did not exceed that expected by chance $\left(\mathrm{df}=5 ; Q_{4}=3.89, p=0.56 ; I^{2}=0\right)$. The associated Funnel Plot was reasonably symmetrical (Figure 4), the FailSafe $\mathrm{N}$ for remission rates was 15, and Egger's regression intercept was $0.33(\mathrm{df}=4 ; t=0.33$; two-tailed $p=0.76)$, also suggesting a low risk of publication bias. Moreover, no RCT was trimmed in the Duval and Tweedie's Trim \& Fill procedure, thus reinforcing the low risk of publication bias.

\section{Acceptability of LF-rTMS Treatment}

Overall, no differences on dropout rates were observed between active LF-rTMS and sham rTMS $(5.3 \%(7 / 132) v s$ $11.28 \% \quad(15 / 133), \quad$ respectively; $\quad \mathrm{OR}=0.53 ; \quad z=-1.23$, $p=0.22$ ) (Figure 5).

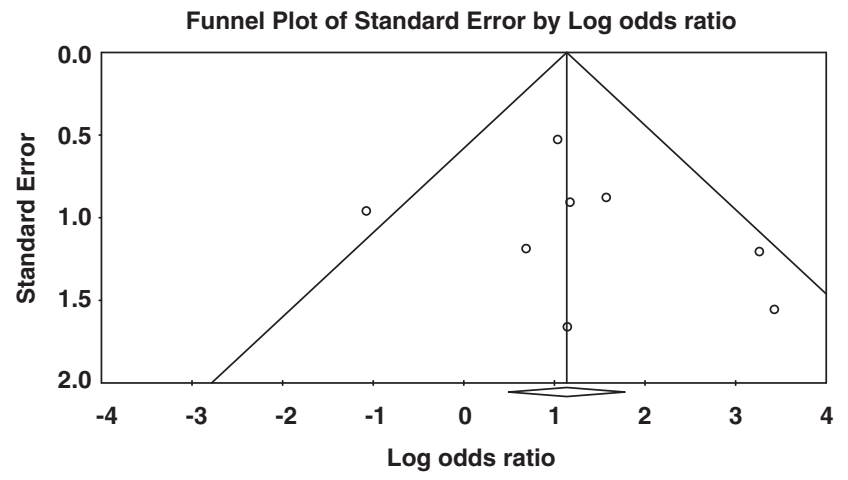

Figure 2 Meta-analysis of LF-rTMS vs Sham rTMS for major depression: funnel plot for studies reporting.

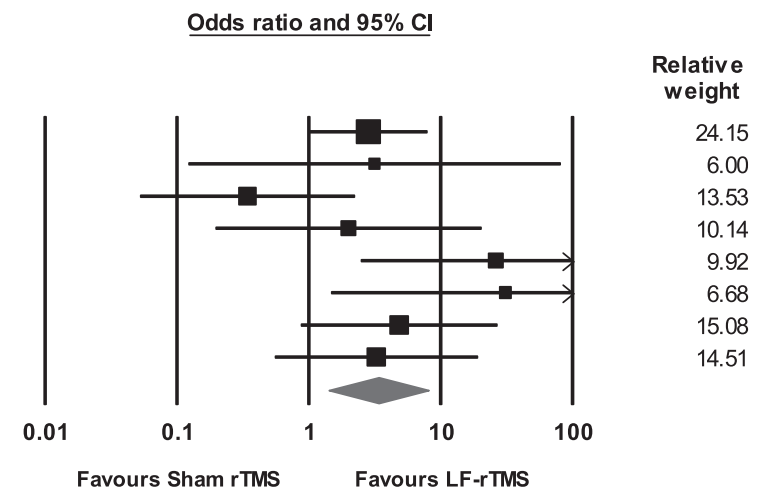

Figure I Meta-analysis of LF-rTMS vs Sham rTMSfor major depression: response rates.

\begin{tabular}{lrrrrrrrr} 
Study name & \multicolumn{9}{c}{ Statistics for each study } \\
& $\begin{array}{r}\text { Odds } \\
\text { ratio }\end{array}$ & $\begin{array}{c}\text { Lower } \\
\text { limit }\end{array}$ & $\begin{array}{c}\text { Upper } \\
\text { limit }\end{array}$ & $\begin{array}{r}\text { Z-Value } \\
\text { p-Value }\end{array}$ & $\begin{array}{c}\text { Active } \\
\text { rTMS }\end{array}$ & $\begin{array}{c}\text { Sham } \\
\text { rTMS }\end{array}$ \\
Kein et al, 1999 & 2.833 & 1.002 & 8.009 & 1.964 & 0.049 & $17 / 35$ & $8 / 32$ \\
Fitzgerald et al, 2003 & 3.154 & 0.121 & 82.165 & 0.691 & 0.490 & $1 / 20$ & $0 / 20$ \\
Hoppner et al, 2003 & 0.343 & 0.052 & 2.261 & -1.112 & 0.266 & $3 / 10$ & $5 / 9$ \\
Kauffman et al, 2004 & 2.000 & 0.194 & 20.614 & 0.582 & 0.560 & $4 / 7$ & $2 / 5$ \\
Januel et al, 2006 & 26.250 & 2.459 & 280.203 & 2.705 & 0.007 & $7 / 11$ & $1 / 16$ \\
Stern et al, 2007 & 31.000 & 1.462 & 657.278 & 2.204 & 0.028 & $5 / 10$ & $0 / 15$ \\
Pallanti et al, 2010 & 4.846 & 0.863 & 27.221 & 1.792 & 0.073 & $7 / 20$ & $2 / 20$ \\
Aguirre et al, 2011 & 3.250 & 0.547 & 19.316 & 1.296 & 0.195 & $6 / 18$ & $2 / 15$ \\
& 3.348 & 1.399 & 8.016 & 2.713 & 0.007 & $50 / 131$ & $20 / 132$
\end{tabular}

Neuropsychopharmacology 


\section{LF-rTMS vs Sham rTMS: Baseline Depression Severity}

No differences on mean baseline depression scores between active and sham rTMS groups were found $(\mathrm{SMD}=0.1$; $z=0.69, p=0.49$ ), thus ruling out illness severity at baseline as a confounding factor. For the associated Forrest Plot please refer to the Supplementary Material.

\section{Sensitivity Analyses}

LF-rTMS protocols delivering $>1200$ magnetic pulses in total were associated with a statistical trend towards higher rates of response to treatment $(\mathrm{OR}=6.9 ; 95 \% \mathrm{CI}=2.39$ $19.92)$ when compared with protocols delivering $\leqslant 1200$ pulses in total $(\mathrm{OR}=1.6 ; 95 \% \mathrm{CI}=0.59-4.39)(Q=3.82$; $\mathrm{df}=1 ; p=0.051)$. However, the total number of sessions and the percentage of the resting motor threshold were not associated with a differential efficacy of LF-rTMS. Additionally, we found no differences in terms of response and remission rates between the RCTs including subjects with unipolar MD only and those including mixed samples of subjects with unipolar and bipolar MD. Finally, response (but not remission) rates were significantly higher for RCTs using LF-rTMS as a monotherapy (OR $=27.94 ; 95 \%$ $\mathrm{CI}=4.3-181.53) v s$ as an augmentation strategy $(\mathrm{OR}=2.32$; $95 \% \mathrm{CI}=1.17-4.6)$ for $\mathrm{MD}(Q=5.99 ; \mathrm{df}=1 ; p=0.014)$. For the associated Forrest Plots please refer to the Supplementary Material.

\section{DISCUSSION}

This is the first meta-analysis assessing the efficacy of LFrTMS to the right DLPFC for MD in terms of response and remission rates. Our results show that this neuromodulation technique is significantly more effective than sham rTMS in producing clinically relevant outcomes (with pooled ORs of 3.35 and 4.76 for response and remission, respectively, and a NNT of 5 for both). Indeed, 3-4 out of 10 depressed subjects receiving LF-rTMS were responders and remitters following a mean of $\sim 13$ sessions, respectively, compared with only about 1 out of 10 of those receiving sham rTMS. Furthermore, we did not find significant differences on dropout rates as well as on baseline depressive symptomatology between active and sham rTMS groups.

This notion is further strengthened by the fact that the observed treatment effect sizes for LF-rTMS are comparable to those reported for several commercially available antidepressants as well as for HF-rTMS. For example, a recent meta-analysis of 122 trials on antidepressants for MD found a pooled drug-placebo rate ratio for response to treatment of $1.42(95 \% \mathrm{CI}=1.38-1.48)$ and a corresponding NNT of $8(95 \% \mathrm{CI}=7.1-9.1)$ (Undurraga and Baldessarini, 2012); our estimate, when converted to rate ratio, is $2.14(95 \% \mathrm{CI}=1.02-4.47)$. Moreover, we have recently shown that the ORs for response and remission after HF-rTMS in MD were $\sim 3$ (Berlim et al, submitted-a). Furthermore, our findings are comparable to those observed in the large and representative Sequenced Treatment Alternatives to Relieve Depression (STAR ${ }^{\star} \mathrm{D}$ ) study (Rush et al, 2006b). More specifically, in the latter, remission rates after lithium carbonate or triiodothyronine augmentation of a second unsuccessful antidepressant course were $20.4 \%$ (Nierenberg et al, 2006). In the current meta-analysis, remission rates following LF-rTMS in depressed individuals who had usually not responded to $\geqslant 2$ antidepressant trials were $34.6 \%$. Such results reinforce the notion that the efficacy of LF-TMS is at least comparable to that of second- or third-line pharmacological strategies for MD. Nevertheless, routine clinical use of rTMS is still limited by its relatively high cost and low availability as well as the lack of clear predictors of treatment response and of data on its medium- to long-term efficacy (George and Post, 2011; Wassermann and Zimmermann, 2012).

As the therapeutic use of LF-rTMS involves several variables, it is possible that the optimum protocol (eg, parameters and duration of stimulation) is yet to be determined. Of relevance, subgroup analyses have shown that the delivery of $>1200$ magnetic pulses in total and the

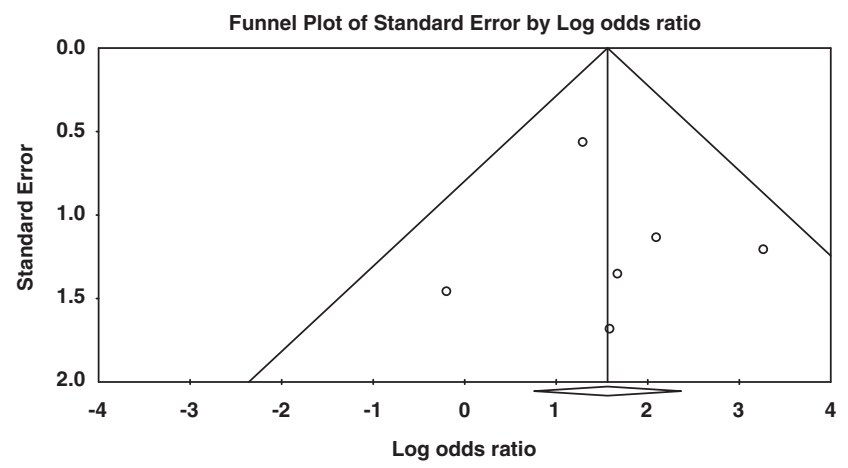

Figure 4 Meta-analysis of LF-rTMS vs Sham rTMS for major depression: funnel plot for studies reporting.

\begin{tabular}{|c|c|c|c|c|c|c|c|}
\hline \multirow[t]{2}{*}{ tudy name } & \multicolumn{5}{|c|}{ Statistics for each study } & \multicolumn{2}{|c|}{ Remitters / Tota } \\
\hline & $\begin{array}{l}\text { Odds } \\
\text { ratio }\end{array}$ & $\begin{array}{c}\text { Lower } \\
\text { limit }\end{array}$ & $\begin{array}{c}\text { Upper } \\
\text { limit }\end{array}$ & -Value & p-Value & $\begin{array}{c}\text { Active } \\
\text { rTMS }\end{array}$ & $\begin{array}{l}\text { Sha } \\
\text { rTM }\end{array}$ \\
\hline & 3.649 & 204 & .064 & 2.287 & 0.022 & $16 / 35$ & 13 \\
\hline auf & & & & & 0.216 & & \\
\hline Januel $\mathrm{C}$ & 26.250 & 2.459 & 280.203 & 2.705 & 0.007 & $7 / 11$ & 11 \\
\hline Stern et & 4.895 & 0.180 & 132.832 & 0.943 & 0.346 & $1 / 10$ & 01 \\
\hline Pallanti et al, 2010 & 8.143 & 0.878 & 75.479 & 1.846 & 0.065 & $6 / 20$ & $1 /$ \\
\hline guirre et al, 2011 & 0.824 & 0.047 & 14.389 & -0.133 & 0.894 & $1 / 18$ & $1 /$ \\
\hline & 4.763 & 2.133 & 10.639 & 3.808 & 0.000 & $35 / 101$ & \\
\hline
\end{tabular}

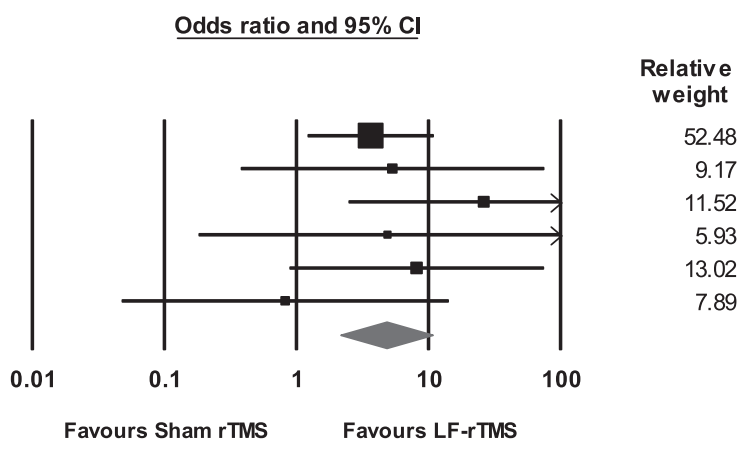

Figure 3 Meta-analysis of LF-rTMS vs Sham rTMS for major depression: remission rates. 
Study name

Kein et al, 1999
Fitzgerald et al, 2003
Hoppner et al, 2003
Kauffman et al, 2004
Januel et al , 2006
Stern et al, 2007
Pallanti et al, 2010
Aguirre et al, 2011

Statistics for each study

\begin{tabular}{|c|c|c|c|}
\hline $\begin{array}{l}\text { Odds } \\
\text { ratio }\end{array}$ & $\begin{array}{c}\text { Lower } \\
\text { limit }\end{array}$ & $\begin{array}{c}\text { Upper } \\
\text { limit }\end{array}$ & Valu \\
\hline 0.441 & 0.038 & 5.113 & -0.655 \\
\hline 1.000 & 0.019 & 52.977 & 0.000 \\
\hline 1.000 & 0.018 & 55.799 & 0.000 \\
\hline 0.692 & 0.011 & 41.777 & -0.176 \\
\hline 0.375 & 0.072 & 1.952 & -1.165 \\
\hline 0.170 & 0.008 & 3.681 & -1.129 \\
\hline 1.000 & 0.019 & 52.977 & 0.000 \\
\hline 2.514 & 0.095 & 66.199 & 0.552 \\
\hline 0.532 & 0.194 & 1.459 & -1.226 \\
\hline
\end{tabular}

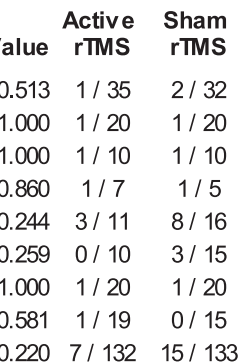

Odds ratio and $95 \% \mathrm{Cl}$

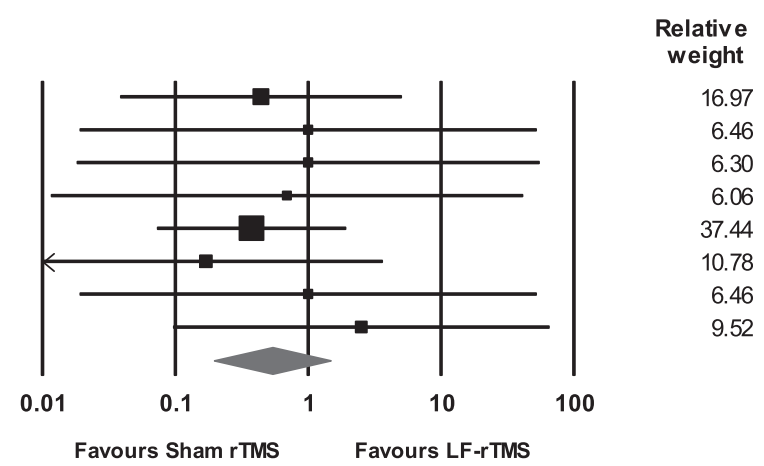

Figure 5 Meta-analysis of LF-rTMS vs Sham rTMS for major depression: dropout rates.

use of rTMS as a monotherapy for MD might be associated with higher rates of response to treatment. Nevertheless, future studies should investigate new ways of enhancing the antidepressant effects of LF-rTMS, such as the identification of more clinically relevant stimulation parameters/protocols (eg, preconditioning paradigms/priming, different rTMS waveforms, frequencies, intensities, number of sessions, brain targets) (Fitzgerald et al, 2008; George and AstonJones, 2010; Peterchev et al, 2011), as well as the use of baseline electrophysiological and/or neuroimaging evaluations to better predict which patients might benefit from LF-rTMS (Arns et al, 2012). Furthermore, novel developments in the field of neuromodulation, such as the H-coil (Levkovitz et al, 2010), might enhance the efficacy of LFrTMS by allowing the direct stimulation of deeper brain structures.

\section{Limitations}

First, although the included RCTs enrolled a relatively small number of depressed subjects, the statistical power to test the summary effects in this meta-analysis was substantially higher than that of any of the primary studies (Borenstein et $a l, 2009)$. Second, the quality of the available sham rTMS conditions is still unresolved (Rosa and Lisanby, 2012), and the use of coil tilting and/or first generation sham coils is clearly not optimal (George and Aston-Jones, 2010; Rossi et al, 2009). In addition, we could not assess the integrity of blinding owing to the absence of this information from all but one of the included RCTs (Fitzgerald et al, 2003) which reported that $42 \%(n=40)$ and $60 \%(n=20)$ of subjects in the active and sham rTMS groups, respectively, were able to correctly guess their treatment allocation before disclosure (at $p>0.05$ ). Furthermore, we have recently conducted a meta-analysis on the integrity of blinding in high-frequency rTMS trials in MD and have showed that after an average of 13 rTMS sessions, 52 and $59 \%$ of subjects receiving active and sham rTMS $(n=396)$, respectively, were able to correctly guess their treatment allocation $(p=0.58)$ (Berlim et al, submitted-b). Third, the most commonly used strategy for locating the DLPFC (ie, the '5-cm method') has been recently criticized for its inaccuracy (Bradfield et al, 2012; Fitzgerald et al, 2009a; Fitzgerald et al, 2009b; Herbsman et al, 2009; Rusjan et al, 2010), and future studies might benefit from neuronavigation approaches (Ruohonen and Karhu, 2010; Schonfeldt-Lecuona et al, 2010). Fourth, we have only examined the efficacy of LF-rTMS at study end, and thus could not estimate the stability of its medium- to long-term antidepressant effects and/or its cost-effectiveness. This was mainly due to the absence of follow-up evaluations on all but two of the included RCTs (Aguirre et al, 2011; Stern et al, 2007). Briefly, Stern et al (2007) reported that the rates of response and remission 2 weeks post-treatment increased from $10-33.3 \%$ (ie, from $1-3$ subjects) and from $50-60 \%$ (ie, from 5-6 subjects) for the LF-rTMS group, respectively, and were maintained at $0 \%$ for the sham rTMS group. Also, Aguirre et al (2011) have shown that the rates of response and remission 4 weeks post-treatment increased from 33.3-38.9\% (ie, from 6-7 subjects) and from $5.5-16.7 \%$ (ie, from 1-3 subjects) for the LF-rTMS group, respectively, and were maintained at the same initial level for the sham rTMS group. Encouragingly, a recent 6-month follow-up study with over 90 depressed subjects has shown that the therapeutic benefits of highfrequency rTMS are durable, and that it can be used for precluding impending relapse (Janicak et al, 2010). Nevertheless, it is clear that future RCTs on rTMS should include longer follow-up periods (eg, >6-12 months), especially considering the labor-intensive and time-consuming nature of rTMS (Wassermann and Zimmermann, 2012). Finally, meta-analyses have been often criticized for the potential of publication bias and for the inclusion of poor-quality trials (Borenstein et al, 2009). In the present study, however, these concerns were addressed by the comprehensive systematic review of the literature and the use of stringent inclusion criteria, and by the objective examination of publication bias and heterogeneity. In particular, the lack of significant heterogeneity among the included RCTs shows that our results are reliable overall. Also, the estimated Fail-Safe Ns for the primary outcome measures varied between 19 and 15 , and we believe that it is unlikely that such a relatively large number of unpublished RCTs with null effects have been either missed by our literature search or were conducted but never published.

\section{CONCLUSION}

The current meta-analysis, which included 263 depressed subjects, provided no evidence that standard antidepressants or HF-rTMS are superior to LF-rTMS applied to the right DLPFC in the treatment of MD. 
Overall, LF-rTMS has a few advantages. For example, it is associated with a significantly lower risk of seizure induction and may even have anti-epileptic properties (Fregni et al, 2006). Therefore, LF-rTMS would be preferred in subjects with risk factors for seizure or with substantial medical comorbidity. Furthermore, LF-rTMS is usually better tolerated than HF-rTMS (which produces a greater degree of local scalp discomfort during stimulation) (Loo et al, 2008), and could thus be potentially offered to a larger number of patients.

Nevertheless, major tasks for future research include the investigation of whether patients with distinct subtypes of MD (eg, bipolar vs unipolar) preferentially respond to this neuromodulation technique, whether its beneficial effects are maintained over time and, especially, how it compares with other rTMS protocols (particularly HF-rTMS). Also, further studies should focus on the search for optimal stimulation parameters as well as on the investigation of the neurobiological underpinnings of the effect of LF-rTMS.

\section{DISCLOSURE}

Dr Daskalakis received external funding through Neuronetics and Brainsway, Aspect Medical and a travel allowance through Pfizer and Merck. Dr Daskalakis has also received speaker funding through Sepracor and served on the advisory board for Hoffmann-La Roche Limited.

\section{REFERENCES}

QuickCalcs - Online Calculators for Scientists - Number needed to treat (NNT) Graphpad Software Vol (2012).

Aguirre I, Carretero B, Ibarra O, Kuhalainen J, Martinez J, Ferrer A et al (2011). Age predicts low-frequency transcranial magnetic stimulation efficacy in major depression. J Affect Disord 130: 466-469.

Allan CL, Herrmann LL, Ebmeier KP (2011). Transcranial magnetic stimulation in the management of mood disorders. Neuropsychobiology 64: 163-169.

APA (1994). Diagnostic and Statistical Manual of Mental Disorders (DSM-IV). 4th edn American Psychiatric Association: Washington, DC.

Arns M, Drinkenburg WH, Fitzgerald PB, Kenemans JL (2012). Neurophysiological predictors of non-response to rTMS in depression. Brain Stimul 5: 569-576.

Berlim MT, Broadbent H, Van den Eynde F. . Blinding integrity in clinical trials of high frequency repetitive transcranial magnetic stimulation (rTMS) for depression: a meta-analysis. Submitted-b.

Berlim MT, Van den Eynde F, Perdomo ST, Daskalakis ZJ. . Response, remission and dropout rates following high frequency repetitive transcranial magnetic stimulation (rTMS) for treating major depression: a systematic review and meta-analysis of randomized, double-blind and sham-controlled trials. Submitted-a.

Borenstein M, Hedges LV, Higgins JPT, Rothstein HR (2009). Introduction to Meta-Analysis. Wiley \& Sons Ltd.: West Sussex, England.

Bradfield NI, Reutens DC, Chen J, Wood AG (2012). Stereotaxic localisation of the dorsolateral prefrontal cortex for transcranial magnetic stimulation is superior to the standard reference position. Aust N Z J Psychiatry 46: 232-239.

Burt T, Lisanby SH, Sackeim HA (2002). Neuropsychiatric applications of transcranial magnetic stimulation: a meta analysis. Int J Neuropsychopharmacol 5: 73-103.
Citrome L (2011). Number needed to treat: what it is and what it isn't, and why every clinician should know how to calculate it. J Clin Psychiatry 72: 412-413.

Cooper H, Hedges LV, Valentine JC (2009). The Handbook of Research Synthesis and Meta-Analysis. Russell Sage Foundation Publications: New York, USA.

Couturier JL (2005). Efficacy of rapid-rate repetitive transcranial magnetic stimulation in the treatment of depression: a systematic review and meta-analysis. J Psychiatry Neurosci JPN 30: 83.

Daskalakis ZJ, Levinson AJ, Fitzgerald PB (2008). Repetitive transcranial magnetic stimulation for major depressive disorder: a review. Can J Psychiatry 53: 555-566.

Deeks JJ (2002). Issues in the selection of a summary statistic for meta-analysis of clinical trials with binary outcomes. Stat Med 21: 1575-1600.

Duval S, Tweedie R (2000). Trim and fill: A simple funnel-plotbased method of testing and adjusting for publication bias in meta-analysis. Biometrics 56: 455-463.

Egger M, Davey Smith G, Schneider M, Minder C (1997). Bias in meta-analysis detected by a simple, graphical test. BMJ 315: 629-634.

Fergusson D, Aaron SD, Guyatt G, Hebert P (2002). Postrandomisation exclusions: the intention to treat principle and excluding patients from analysis. BMJ 325: 652-654.

Fitzgerald PB, Brown TL, Daskalakis ZJ (2002). The application of transcranial magnetic stimulation in psychiatry and neurosciences research. Acta Psychiatr Scand 105: 324-340.

Fitzgerald PB, Brown TL, Marston NA, Daskalakis ZJ, De Castella A, Kulkarni J (2003). Transcranial magnetic stimulation in the treatment of depression: a double-blind, placebo-controlled trial. Arch Gen Psychiatry 60: 1002-1008.

Fitzgerald PB, Hoy K, McQueen S, Herring S, Segrave R, Been G et al (2008). Priming stimulation enhances the effectiveness of low-frequency right prefrontal cortex transcranial magnetic stimulation in major depression. J Clin Psychopharmacol 28: 52-58.

Fitzgerald PB, Hoy KE, McQueen S, Maller JJ, Herring S, Segrave R et al (2009a). A randomized trial of rTMS targeted with MRI based neuro-navigation in treatment-resistant depression. Neuropsychopharmacology 34: 1255-1262.

Fitzgerald PB, Maller JJ, Hoy KE, Thomson R, Daskalakis ZJ (2009b). Exploring the optimal site for the localization of dorsolateral prefrontal cortex in brain stimulation experiments. Brain Stimul 2: 234-237.

Fregni F, Otachi PT, Do Valle A, Boggio PS, Thut G, Rigonatti SP et al (2006). A randomized clinical trial of repetitive transcranial magnetic stimulation in patients with refractory epilepsy. Ann Neurol 60: 447-455.

Fregni F, Pascual-Leone A (2007). Technology insight: noninvasive brain stimulation in neurology-perspectives on the therapeutic potential of rTMS and tDCS. Nat Clin Pract Neurol 3: 383-393.

George MS, Aston-Jones G (2010). Noninvasive techniques for probing neurocircuitry and treating illness: vagus nerve stimulation (VNS), transcranial magnetic stimulation (TMS) and transcranial direct current stimulation (tDCS). Neuropsychopharmacology 35: 301-316.

George MS, Padberg F, Schlaepfer TE, O’Reardon JP, Fitzgerald PB, Nahas ZH et al (2009). Controversy: Repetitive transcranial magnetic stimulation or transcranial direct current stimulation shows efficacy in treating psychiatric diseases (depression, mania, schizophrenia, obsessive-complusive disorder, panic, posttraumatic stress disorder). Brain Stimul 2: 14-21.

George MS, Post RM (2011). Daily left prefrontal repetitive transcranial magnetic stimulation for acute treatment of medication-resistant depression. Am J Psychiatry 168: 356-364. 
George MS, Wassermann EM, Williams WA, Callahan A, Ketter TA, Basser P et al (1995). Daily repetitive transcranial magnetic stimulation (rTMS) improves mood in depression. Neuroreport 6: $1853-1856$.

Gross M, Nakamura L, Pascual-Leone A, Fregni F (2007). Has repetitive transcranial magnetic stimulation (rTMS) treatment for depression improved? A systematic review and meta-analysis comparing the recent vs. the earlier rTMS studies. Acta Psychiatr Scand 116: 165-173.

Hamilton M (1960). A rating scale for depression. J Neurol Neurosurg Psychiatry 23: 56-62.

Herbsman T, Avery D, Ramsey D, Holtzheimer P, Wadjik C, Hardaway $\mathrm{F}$ et al (2009). More lateral and anterior prefrontal coil location is associated with better repetitive transcranial magnetic stimulation antidepressant response. Biol Psychiatry 66: $509-515$.

Herrmann LL, Ebmeier KP (2006). Factors modifying the efficacy of transcranial magnetic stimulation in the treatment of depression: a review. J Clin Psychiatry 67: 1870-1876.

Higgins JPT, Green S (2008). Cochrane Handbook for Systematic Reviews of Interventions. 1st edn John Wiley \& Sons Ltd.: West Sussex, England.

Hoppner J, Schulz M, Irmisch G, Mau R, Schlafke D, Richter J (2003). Antidepressant efficacy of two different rTMS procedures. High frequency over left versus low frequency over right prefrontal cortex compared with sham stimulation. Eur Arch Psychiatry Clin Neurosci 253: 103-109.

Huf W, Kalcher K, Pail G, Friedrich ME, Filzmoser P, Kasper S (2011). Meta-analysis: fact or fiction? How to interpret metaanalyses. World J Biol Psychiatry 12: 188-200.

Janicak PG, Nahas Z, Lisanby SH, Solvason HB, Sampson SM, McDonald WM et al (2010). Durability of clinical benefit with transcranial magnetic stimulation (TMS) in the treatment of pharmacoresistant major depression: assessment of relapse during a 6-month, multisite, open-label study. Brain Stimul 3: $187-199$.

Janicak PG, O’Reardon JP, Sampson SM, Husain MM, Lisanby SH, Rado JT et al (2008). Transcranial magnetic stimulation in the treatment of major depressive disorder: a comprehensive summary of safety experience from acute exposure, extended exposure, and during reintroduction treatment. J Clin Psychiatry 69: $222-232$.

Januel D, Dumortier G, Verdon CM, Stamatiadis L, Saba G, Cabaret $\mathrm{W}$ et al (2006). A double-blind sham controlled study of right prefrontal repetitive transcranial magnetic stimulation (rTMS): therapeutic and cognitive effect in medication free unipolar depression during 4 weeks. Prog Neuropsychopharmacol Biol Psychiatry 30: 126-130.

Kauffmann CD, Cheema MA, Miller BE (2004). Slow right prefrontal transcranial magnetic stimulation as a treatment for medication-resistant depression: a double-blind, placebo-controlled study. Depress Anxiety 19: 59-62.

Klein E, Kreinin I, Chistyakov A, Koren D, Mecz L, Marmur S et al (1999). Therapeutic efficacy of right prefrontal slow repetitive transcranial magnetic stimulation in major depression: a doubleblind controlled study. Arch Gen Psychiatry 56: 315-320.

Kozel FA, George MS (2002). Meta-analysis of left prefrontal repetitive transcranial magnetic stimulation (rTMS) to treat depression. J Psychiatr Pract 8: 270-275.

Lam RW, Chan P, Wilkins-Ho M, Yatham LN (2008). Repetitive transcranial magnetic stimulation for treatment-resistant depression: a systematic review and metaanalysis. Can J Psychiatry 53: 621-631.

Levkovitz Y, Harel EV, Roth Y, Braw Y, Most D, Katz LN et al (2010). Deep transcranial magnetic stimulation over the prefrontal cortex: evaluation of antidepressant and cognitive effects in depressive patients. Brain Stimulation 4: 188-200.
Loo CK, McFarquhar TF, Mitchell PB (2008). A review of the safety of repetitive transcranial magnetic stimulation as a clinical treatment for depression. Int J Neuropsychopharmacol 11: 131-147.

Marangell LB, Martinez M, Jurdi RA, Zboyan H (2007). Neurostimulation therapies in depression: a review of new modalities. Acta Psychiatr Scand 116: 174-181.

Martin JL, Barbanoj MJ, Schlaepfer TE, Clos S, Perez V, Kulisevsky J et al (2002). Transcranial magnetic stimulation for treating depression. Cochrane Database Syst Rev: CD003493.

Martin JL, Barbanoj MJ, Schlaepfer TE, Thompson E, Perez V, Kulisevsky J (2003). Repetitive transcranial magnetic stimulation for the treatment of depression. Systematic review and metaanalysis. Br J Psychiatry 182: 480-491.

Maxwell SE, Kelley K, Rausch JR (2008). Sample size planning for statistical power and accuracy in parameter estimation. Annu Rev Psychol 59: 537-563.

McNamara B, Ray JL, Arthurs OJ, Boniface S (2001). Transcranial magnetic stimulation for depression and other psychiatric disorders. Psychol Med 31: 1141-1146.

Montgomery SA, Asberg M (1979). A new depression scale designed to be sensitive to change. Br J Psychiatry 134: 382-389.

Nierenberg AA, Fava M, Trivedi MH, Wisniewski SR, Thase ME, McGrath PJ et al (2006). A comparison of lithium and T(3) augmentation following two failed medication treatments for depression: a STAR ${ }^{\star} \mathrm{D}$ report. Am J Psychiatry 163: 1519-1530 quiz 1665.

Pallanti S, Bernardi S, Di Rollo A, Antonini S, Quercioli L (2010). Unilateral low frequency versus sequential bilateral repetitive transcranial magnetic stimulation: is simpler better for treatment of resistant depression? Neuroscience 167: 323-328.

Peterchev AV, Wagner TA, Miranda PC, Nitsche MA, Paulus W, Lisanby SH et al (2011). Fundamentals of transcranial electric and magnetic stimulation dose: definition, selection, and reporting practices. Brain Stimul 5: 435-453.

Riley RD, Higgins JP, Deeks JJ (2011). Interpretation of random effects meta-analyses. BMJ 342: d549.

Rosa MA, Lisanby SH (2012). Somatic treatments for mood disorders. Neuropsychopharmacology 37: 102-116.

Rosenthal R (1979). The File Drawer problem and tolerance for null results. Psychol Bull 86: 638-641.

Rossi S, Hallett M, Rossini PM, Pascual-Leone A (2009). Safety, ethical considerations, and application guidelines for the use of transcranial magnetic stimulation in clinical practice and research. Clin Neurophysiol 120: 2008-2039.

Ruohonen J, Karhu J (2010). Navigated transcranial magnetic stimulation. Neurophysiol Clin 40: 7-17.

Rush AJ, Kraemer HC, Sackeim HA, Fava M, Trivedi MH, Frank E et al (2006a). Report by the ACNP Task Force on response and remission in major depressive disorder. Neuropsychopharmacology 31: 1841-1853.

Rush AJ, Trivedi MH, Wisniewski SR, Nierenberg AA, Stewart JW, Warden D et al (2006b). Acute and longer-term outcomes in depressed outpatients requiring one or several treatment steps: a STAR*D Report. Am J Psychiatry 163: 1905-1917.

Rusjan PM, Barr MS, Farzan F, Arenovich T, Maller JJ, Fitzgerald $\mathrm{PB}$ et al (2010). Optimal transcranial magnetic stimulation coil placement for targeting the dorsolateral prefrontal cortex using novel magnetic resonance image-guided neuronavigation. Hum Brain Mapp 31: 1643-1652.

Schlaepfer TE, Kosel M, Nemeroff CB (2003). Efficacy of repetitive transcranial magnetic stimulation (rTMS) in the treatment of affective disorders. Neuropsychopharmacology 28: 201-205.

Schonfeldt-Lecuona C, Lefaucheur JP, Cardenas-Morales L, Wolf RC, Kammer T, Herwig U (2010). The value of neuronavigated rTMS for the treatment of depression. Neurophysiol Clin 40: 37-43. 
Schutter DJ (2009). Antidepressant efficacy of high-frequency transcranial magnetic stimulation over the left dorsolateral prefrontal cortex in double-blind sham-controlled designs: a meta-analysis. Psychol Med 39: 65-75.

Schutter DJ (2010). Quantitative review of the efficacy of slowfrequency magnetic brain stimulation in major depressive disorder. Psychol Med 40: 1789-1795.

Slotema CW, Blom JD, Hoek HW, Sommer IE (2010). Should we expand the toolbox of psychiatric treatment methods to include Repetitive Transcranial Magnetic Stimulation (rTMS)? A metaanalysis of the efficacy of rTMS in psychiatric disorders. J Clin Psychiatry 71: 873-884.

Speer AM, Benson BE, Kimbrell TK, Wassermann EM, Willis MW, Herscovitch P et al (2009). Opposite effects of high and low frequency rTMS on mood in depressed patients: relationship to baseline cerebral activity on PET. J Affect Disord 115: 386-394.

Speer AM, Kimbrell TA, Wassermann EM, DR J, Willis MW, Herscovitch $\mathrm{P}$ et al (2000). Opposite effects of high and low frequency rTMS on regional brain activity in depressed patients. Biol Psychiatry 48: 1133-1141.

Stern WM, Tormos JM, Press DZ, Pearlman C, Pascual-Leone A (2007). Antidepressant effects of high and low frequency repetitive transcranial magnetic stimulation to the dorsolateral prefrontal cortex: a double-blind, randomized, placebo-controlled trial. J Neuropsychiatry Clin Neurosci 19: 179-186.

Undurraga J, Baldessarini RJ (2012). Randomized, placebocontrolled trials of antidepressants for acute major depression: thirty-year meta-analytic review. Neuropsychopharmacology 37: 851-864.

Wassermann EM, Zimmermann T (2012). Transcranial magnetic brain stimulation: therapeutic promises and scientific gaps. Pharmacol Ther 133: 98-107.

WHO (1992). The ICD-10 Classification of Mental and Behavioural Disorders: Clinical Descriptions and Diagnostic Guidelines. 10th edn World Health Organization: Geneva, Switzerland.

Supplementary Information accompanies the paper on the Neuropsychopharmacology website (http://www.nature.com/npp) 